\title{
A Multi(two)-Nozzle Cable-Driven Parallel Robot For 3D Printing Building Construction: Path Optimization and Vibration Analysis
}

\section{Sy Nguyen-Van}

Thai Nguyen University of Technology

Kwan-Woong Gwak ( $\sim$ kwgwak@sejong.ac.kr)

Sejong University https://orcid.org/0000-0001-9170-3139

\section{Research Article}

Keywords: 3D printing building construction, Multi-nozzle cable-driven parallel robots, Finite element method, Free vibration analysis, Genetic algorithm, Particle swarm optimization, Differential evolution, NURBS, Path optimization

Posted Date: November 2nd, 2021

DOI: https://doi.org/10.21203/rs.3.rs-1036880/v1

License: (c) (i) This work is licensed under a Creative Commons Attribution 4.0 International License. Read Full License

Version of Record: A version of this preprint was published at The International Journal of Advanced Manufacturing Technology on March 3rd, 2022. See the published version at https://doi.org/10.1007/s00170-022-08919-5. 


\title{
A multi(two)-nozzle cable-driven parallel robot for 3D printing building construction: path optimization and vibration analysis
}

\author{
Sy Nguyen-Van ${ }^{1}$, Kwan-Woong Gwak ${ }^{2 *}$ \\ 1 Department of Mechanical Engineering, Thai Nguyen University of Technology, Thai Nguyen, Vietnam \\ 2 Department of Mechanical and Aerospace Engineering, Sejong University, 209 Neungdong-Ro, Gwangjin-gu, Seoul, 05006, Korea \\ \# Corresponding Author / E-mail: kwgwak@sejong.ac.kr, TEL: +82-2-3408-3785
}

KEYWORDS : 3D printing building construction, Multi-nozzle cable-driven parallel robots, Finite element method, Free vibration analysis, Genetic algorithm, Particle swarm optimization, Differential evolution, NURBS, Path optimization

\begin{abstract}
This paper proposes a multi-nozzle cable-driven parallel robot for $3 D$ printing building construction. This system has two independently moving nozzles mounted on the existing printing head. The printing time can be reduced dramatically with this system as the travel path of the printing head can be reduced to almost half thanks to those two nozzles that print almost half of the printing contour. To fully take advantage of two nozzle structures effectively, the path of the printing head is optimized to secure the minimum travel length of both the printing head and two nozzles. The smoothness of the optimal path is secured by applying the non-uniform rational B-splines (NURBS). In addition, free vibration of the proposed CDPR printer's structure is analyzed to improve the printing quality and help the control of the proposed CDPR plain by using a finite element formulation of cables of the proposed robot.
\end{abstract}

\section{Introduction}

In recent years, 3D-printing technology has been creating lots of applications in many industries, from mechanical manufacturing to medical applications. 3D-printing has now expanded its application area even to the building construction. Due to the large-scale of the required work in construction, a conventional 3D-printer based on the gantry or $x-y-z$ Cartesian mechanism might not be suitable because they are heavy, has limitation in dimension expansion and are expensive. Thus cable-driven parallel robots (called CDPRs) have been proposed for 3D printing building construction [1]-[5] for their benefits in terms of large workspace, low cost, lightweight, flexible reconfiguration, and easy transportation [2]. Along with such benefits, CDPRs could be more competitive for 3D printing building constructions if construction time can be reduced more as the construction time is a key factor for the fulfillment of the construction deadline and the cost. Reducing the time for the 3D printing building construction can be achieved by several options such as using rapidsolidifying printing material, reducing the printing path length and etc. In this research, we focus on reducing the printing path length approach. Also, as a viable option for path length reduction, we propose a CDPR with a multi-nozzle printing head. With the multi-nozzle printing head, path length of the printing head can be reduced dramatically as each nozzle covers the portion of the required printing path.

Considering the fact that the contour of the most buildings to be printed has a closed-shape, the number of nozzles are selected as two in this research. To the best knowledge of the authors, there has been no such multi(two)-nozzle CDPRs reported in the literature.

As can be imagined, applying extra nozzles to the existing single nozzle printing head to print certain portions of the building component would require a change in the whole process of $3 \mathrm{D}$ printing including 
the path planning. Especially for printing an asymmetric part using two nozzles, it requires independent motion control of each nozzles to print the object. Hence, there is a need for a specific path planning for the integrated system which has multiple nozzles working together [6].

Typical path planning aims to find a collision-free path from a starting point to a target point, and to satisfy some criteria such as distance, time, or energy. Among them, the distance is one of the most commonly adopted criteria [7].

To find the optimal path for the two-nozzle CDPR, the following three constraints are considered: (1) The path of the printing head should line in the middle of the contour of the parts to be printed to minimize the time required for the movement of both nozzles. This will minimize the difference between the path lengths of two nozzles, thus both nozzles move the same length as much as possible which would make the motion control of two nozzles plain (2) The path length of the printing head should be minimum to minimize the printing time. (3) the path shall be as smooth as possible and at least satisfy the continuity so that it can be implemented physically. The first two constraints are related not only with time, but also with the energy of motion control system of the CDPR with two-nozzle printing head. These three constraints form the basis for the objective function for the path optimization problem.

To find out the optimal path of the printing head, optimization algorithms need to be determined to solve such objective functions for the path planning. For path planning, numerous optimization algorithms have been proposed and they can be divided into two groups: (1) classical methods; (2) evolutionary methods. Some of the most used classical methods are Cell Decomposition (CD), Potential Field (PF), RoadMap, Subgoal Network (SN) [8]. However, the main drawbacks of the classical methods are that they are difficult to find an optimum path for the complex system [9]. In addition, the time for the determination of feasible collision-free path increased and these algorithms might be trapped in the local optimal solution [7]. Hence, evolutionary algorithms, such as Genetic Algorithm (GA) [10], [11], Particle Swarm Optimization (PSO) [12], [13], and Differential Evolution (DE) [14], [15], which randomly find solutions in a given search space can be reasonable alternatives for complex problems. Hence, GA, DE, and PSO algorithms are applied in this research to find the optimal paths of the printing head of the two-nozzle CDPR 3D printer.

For the smooth path generation, the non-uniform rational B-Spline (NURBS) is applied. The NURBS curves have been noticed by research communities for robotic path planning [16]-[18] because it has a high level of flexibility and a great capacity to produce natural smooth curves [18]. A NURBS curve is defined by its order, a set of weighted control points, and a knot vector.

Regarding the multi(two)-nozzle CDPRs for 3D printing building construction, in addition to the path planning, there is another important issue that needs to be handled. Due to the inevitably flexible characteristics of cables, the multi(two)-nozzle CDPRs for 3D printing building construction are highly capable of vibrating in both axial and transversal directions [19]. It is obvious that the vibration gives an adverse effect on the performance of the multi(two)-nozzle CDPR. Hence, vibration analysis for the multi(two)-nozzle CDPR for 3D printing building construction should be conducted. In this research, the finite element method which was reported in [5] is applied to study the vibration of the multi-nozzle CDPR 3D printer for the first time. The natural frequency which is a good parameter to evaluate the stiffness and dynamic performance of CDPRs are computed and its validity is confirmed through the comparison with those of commercial software, SAP2000.

\section{The two nozzle cable driven parallel robot}

This section presents a novel conceptual design of the multi(two)nozzle CDPR which could reduce the printing-time dramatically. In

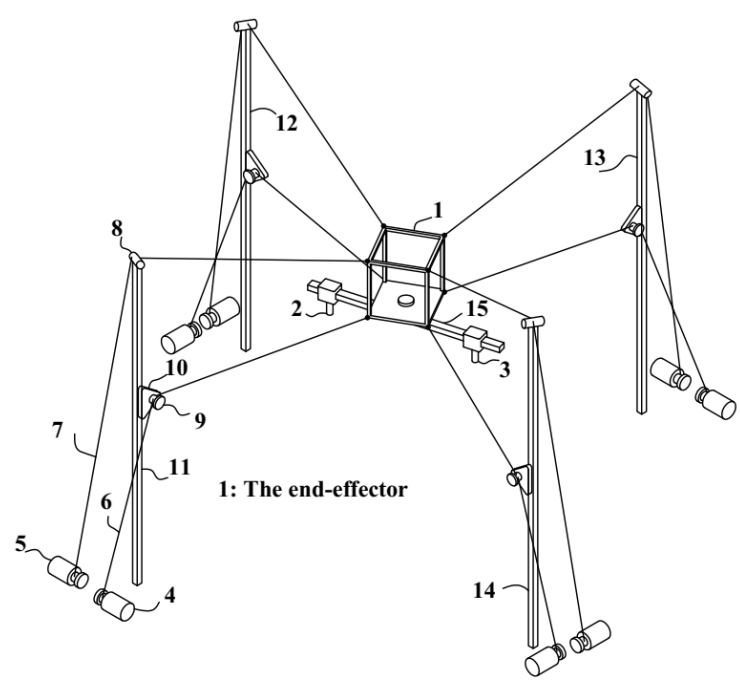

Fig. 1 The proposed multi(two)-nozzle CDPR 3D printer.

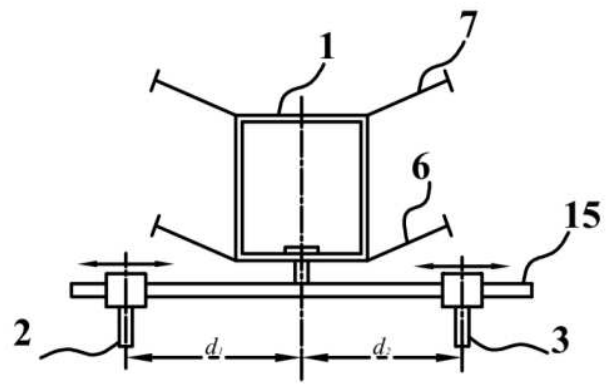

Fig. 2 A general scheme of two nozzles mounted on the printing head (Front view). 


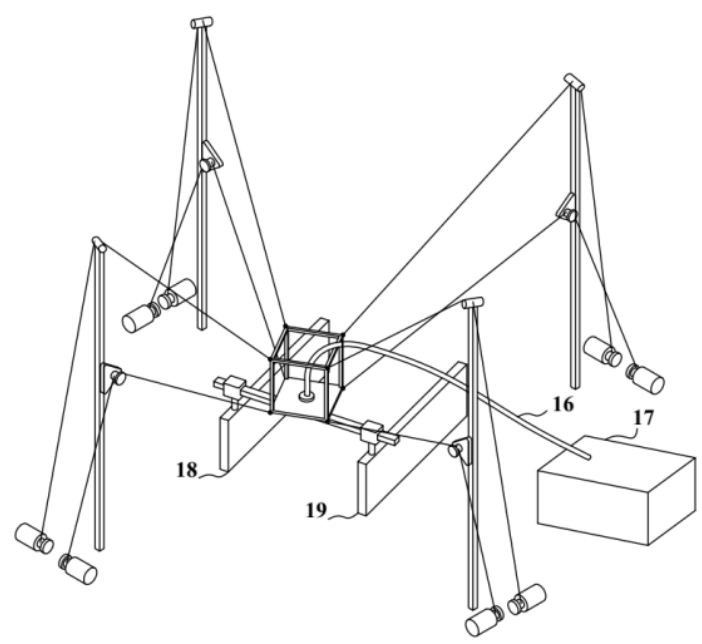

Fig. 3 A simplified view of the proposed CDPR of Fig. 1 being used to print symmetric part of the building construction with two fixed nozzles.

addition, the dynamic and kinematic analysis of the printing head and nozzles are also derived.

\subsection{Conceptual design}

A multi-nozzle CDPR for constructional 3D printing saving printingtime and cost of construction simultaneously is proposed in Fig. 1. The number of nozzles are selected as two considering the fact that most of the contour of the buildings to be printed are closed-shape. Two-nozzles are attached to the printing head as shown in Fig. 1. In the figure, an embodiment of a fully constrained two-nozzle cable-driven parallel robot includes a printing head $\mathbf{1}$, two-nozzles $\mathbf{2}$ and $\mathbf{3}$, the sliding bar that extends the nozzle length $\mathbf{1 5}$, four vertical support columns 11, 12, 13, 14, drums 8 and 9 mounted on each vertical columns, lower cables $\mathbf{6}$ and upper cables 7. The pair of cables $\mathbf{6}$ and $\mathbf{7}$ are twisted around the drums 9 and 8 , respectively, and are driven by actuators $\mathbf{4}$ and $\mathbf{5}$. These motors are placed outside the workspace and are placed on the ground. Each drum 9 is mounted on a bracket $\mathbf{1 0}$. The bracket can move up and down along the vertical support column. Thus, it makes the present embodiment reconfigurable and helps avoid the interference between its system and building parts being printed. Overall, this system has 8 cables to suspend the printing head. The poses of the printing head and the nozzles are adjusted by changing the lengths of the cables routed through drums.

Fig. 2 shows a front view of the two nozzles $\mathbf{2}$ and $\mathbf{3}$. These nozzles can move along the axis of the sliding bar $\mathbf{1 5}$ and can move independently to print the asymmetric parts at the same time. The sliding bars 15 can rotate around the printing head to flexibly print various printing shapes. In the case of printing the same-shaped multiple parts or symmetric parts like the walls 18 and 19 as in Fig.3, the lengths of the nozzles are fixed. In contrast, for printing an un-

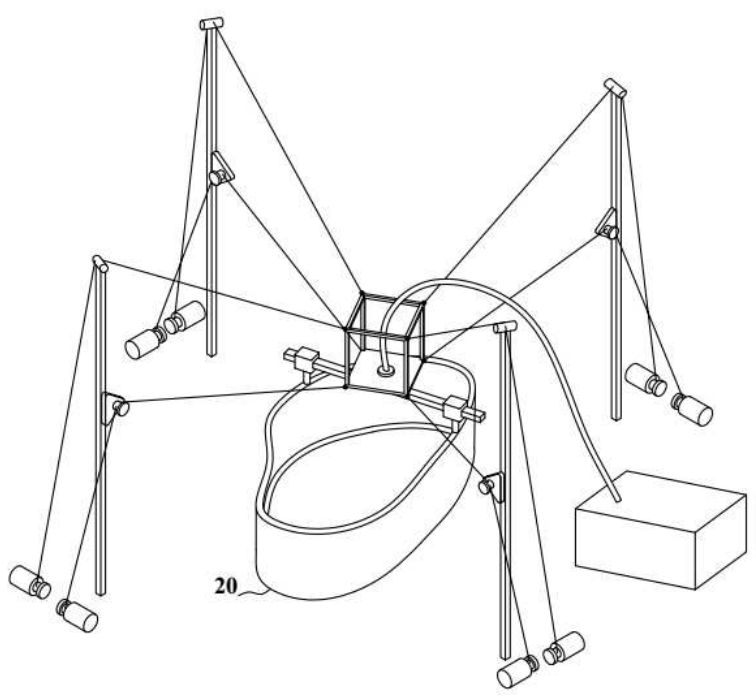

Fig. 4 A simplified view of the proposed CDRP of Fig. 1 being used to print an asymmetric shape of the building construction with two moving nozzles.

symmetric part $\mathbf{2 0}$ as shown in Fig. 4, each nozzles are controlled independently and differently. Hence, the proper path of the printing head is needed to be determined to control both nozzles independently and differently. Note that $\mathbf{1 6}$ and $\mathbf{1 7}$ in Fig. 3, represent a supply tube and a pump that feed the $3 \mathrm{D}$ constructional printing materials such as concrete or foam.

\subsection{Dynamic equation of the printing head}

This section presents the dynamic equation of the printing head. The dynamic behavior of the printing head can be considered as the dynamics of the end-effector of the general cable robot. As reported in [20], a general scheme of the end effector can be shown as in Fig. 5. The printing head is driven by $\mathrm{n}$ cables. The $i t h$ cable is attached on the base and the end effector at $A_{i}$ and $B_{i}$, respectively. The vector $\mathbf{l}_{i}$ denotes the cable $i$ th and its norm is the length of the cable. $S_{\mathbf{i}}$ is the unit vector of the cable ith. With the global frame $\mathrm{O}_{0}$, the positions of $\mathrm{A}_{i}$ and the centroid of the end effector (the point $\mathrm{C}$ ) are vector $\mathbf{a}_{\mathbf{i}}$ and $\mathbf{p}$, respectively. With the reference frame $\mathrm{O}_{1}$ attached at point $\mathrm{C}$ and $\mathbf{b}_{\mathbf{i}}$ being the position vector of the point $B_{i}$, position of the centroid of the end effector can be written as follows:

$$
\mathbf{p}=\mathbf{a}_{\mathrm{i}}+\mathbf{l}_{\mathrm{i}}-\mathbf{b}_{\mathrm{i}} \quad(i=1,2, \ldots, n)
$$

where, all the vectors are measured with respect to the global frame, $\mathrm{O}_{0}$. The first kinematic equation is given as follows:

$$
\mathbf{l}_{\mathrm{i}}^{2}=\left[\mathbf{p}-\mathbf{a}_{\mathrm{i}}+\mathbf{b}_{\mathrm{i}}\right]^{\mathrm{T}}\left[\mathbf{p}-\mathbf{a}_{\mathrm{i}}+\mathbf{b}_{\mathrm{i}}\right]
$$

Next, differentiation of the previous equation with respect to time and arranging the nth equations into a matrix form yields: 


$$
\mathbb{E}=\mathbf{J t}
$$

Where,

$$
\begin{gathered}
\mathbf{E}=\left[\begin{array}{llll}
\mathbb{L} & \& & \ldots & \ell_{n}^{\&}
\end{array}\right]^{T} \\
\mathbf{J}=\left[\begin{array}{cccl}
\mathbf{S}_{1} & \mathbf{S}_{2} & \ldots & \mathbf{S}_{\mathrm{n}} \\
\mathbf{b}_{1} \times \mathbf{S}_{1} & \mathbf{b}_{2} \times \mathbf{S}_{2} & \ldots & \mathbf{b}_{\mathrm{n}} \times \mathbf{S}_{\mathrm{n}}
\end{array}\right]^{T} \\
\mathbf{t}=\left[\begin{array}{c}
\mathcal{Q P} \\
\boldsymbol{\omega}^{T}
\end{array}\right]
\end{gathered}
$$

In these above equations $p \&$ and $\boldsymbol{\omega}$ are the linear velocity vector of point $\mathrm{C}$ and the angular velocity vector of the end effector, respectively; $\mathbf{J}$ is the [ $\mathrm{n} \times$ 6] Jacobian matrix of the cable robot. Thus, the general dynamic equation of cable robot can be presented as follows [19]:

$$
\begin{aligned}
& {\left[\begin{array}{cc}
m \mathbf{I}_{3 \times 3} & \mathbf{0}_{3 \times 3} \\
\mathbf{0}_{3 \times 3} & \mathbf{I}_{P}
\end{array}\right]\left[\begin{array}{c}
\mathbf{f} \\
\boldsymbol{\alpha}
\end{array}\right]+} \\
& +\left[\begin{array}{c}
\mathbf{0}_{3 \times 1} \\
\boldsymbol{\omega} \times \mathbf{I}_{P} \times \boldsymbol{\omega}
\end{array}\right]+\left[\begin{array}{c}
-m \mathbf{g} \\
\mathbf{0}_{3 \times 1}
\end{array}\right]+\left[\begin{array}{l}
\mathbf{f}_{e} \\
\boldsymbol{\tau}_{e}
\end{array}\right]=\mathbf{J}^{T} \mathbf{T}
\end{aligned}
$$

Where, $m$ is the mass of the end effector; IP is the inertia tensor of the end effector about point $\mathrm{C}$ in the frame $\mathrm{O}_{0} ; \mathbf{I}_{3 \times 3}$ is the $3 \times 3$ identity matrix; $\mathbf{g}$ is the gravity acceleration vector; $\mathbf{f}_{\mathrm{e}}$ and $\tau_{\mathrm{e}}$ are the external force and moment vector applied to the printing head, respectively; $\mathbf{T}$ is the vector of cable forces. It should be noted that cables cannot be compressed, and the vector of cable force must be satisfied with the following force closure equation:

$$
\mathbf{J}^{T} \mathbf{T}=\mathbf{w}
$$

where $\mathbf{w}$ is the resultant wrench applied at the end effector defined as follows.

$$
\mathbf{w}=\left[\begin{array}{c}
-m \mathbf{g} \\
\mathbf{0}_{3 \times 1}
\end{array}\right]+\left[\begin{array}{l}
\mathbf{f}_{e} \\
\boldsymbol{\tau}_{e}
\end{array}\right]
$$

The determination of the cable tension is a key to the operation of the CDPR. The end-effector of the proposed CDPR operates with eight cables but the motion of the end-effector is governed by only six dynamic equations of motion defined in Eq. (4). ThiZs implies an under-determined problem hence eight cable tensions need to be solved by some form of optimization algorithms. Hence, a nonlinear programming form optimization problem is posed as follows for the determination of the cable tension distribution:

$$
\begin{gathered}
\text { minimize: } \frac{1}{2} \mathbf{T}^{T} \mathbf{C T}+\mathbf{c}^{T} \mathbf{T} \\
\text { subject to: } T_{\min } \leq T_{i} \leq T_{\max } \\
\mathbf{J}^{T} \mathbf{T}=\mathbf{W}
\end{gathered}
$$

Where $\mathbf{C}$ and $\mathbf{c}$ are the weighting factor for the objective function in Eq.(7) and $T_{\max }$ and $T_{\min }$ are the minimum and the maximum tension allowed to cables, respectively.

Note that 2-norm is used as the objective function of the quadratic programming because discontinuities could result when 1-norm is used as reported in [2].

The weighting factors are set as follows: $\mathbf{C}=\mathbf{I}$ for the quadratic part and $\mathbf{c}=\mathbf{0}$ for nullifying the linear part.

\subsection{Fixed two-nozzles mounted to the main printing head}

When the two additional nozzles are fixed at the printing head to print the same-shaped objects, these nozzles and the printing head become one rigid body. Then, two nozzles will result in the external forces (f1) and external moments $(\tau 1)$ to the printing head by the following two equations.

$$
\begin{aligned}
& f_{1}=\left(m_{1}+m_{2}\right) g \\
& \tau_{1}=m_{1} g d_{1}-m_{2} g d_{2}
\end{aligned}
$$

Where, $m_{1}$ and $m_{2}$ are the mass of the two nozzles, respectively; $d_{1}$ and $\mathrm{d}_{2}$ are the relative positions of the two with respect to the printing head, respectively.

\subsection{Moving-nozzles mounted to the main printing head}

Generally, it is assumed that the end-effector of a CDPR for the 3D printing construction moves with small velocity and constant speed in most cases. Hence we can neglect the dynamic effect of the printing head on the motion of the moving nozzles. The moving nozzles exert the external forces $\left(f_{1}\right)$ and external moments $\left(\tau_{1}\right)$ to the printing head as in Eq. (10) and they can be controlled by two linear motors attached to the printing head. As the sliding bar on which two nozzles slides is assumed to have very low friction, the dynamic equation of two moving nozzles are given as follows:

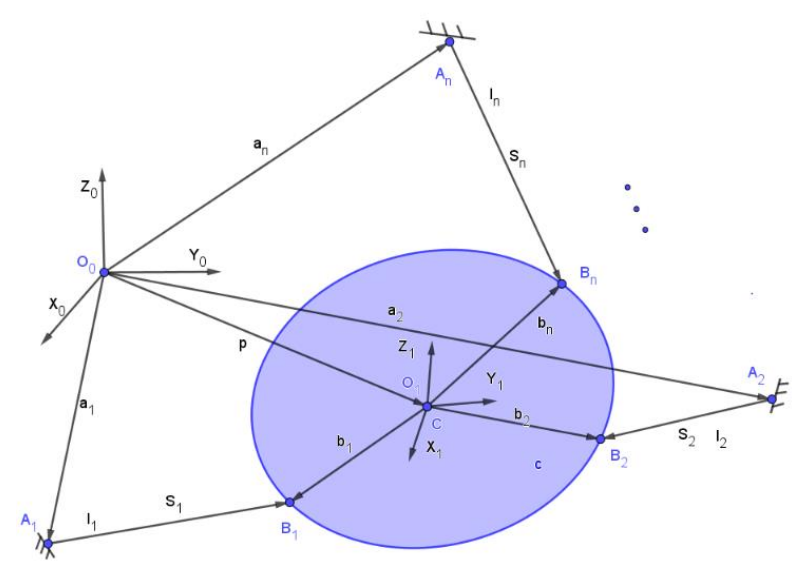

Fig. 5 A general scheme of the printing head [19]. 


$$
\begin{aligned}
& m_{1} \&_{1}=F_{1} \\
& m_{2} \&_{2}=F_{2}
\end{aligned}
$$

Where, and are the acceleration of the two nozzles; $F_{1}$ and $F_{2}$ are the applied forces by the nozzle-driving actuators to the two nozzles.

\section{Free vibration analysis}

This section presents a finite element formulation to analyze the vibration of the proposed system. Then, some numerical analyses are performed to investigate its vibration behavior.

\subsection{Cable formulation}

For vibration analysis, cables attached between drums and the printing head are divided into small cable elements. Each cable element contains two nodes, $\mathrm{i}$ and $\mathrm{j}$, with the length of $\mathrm{l}_{\mathrm{ij}}$ and the applied tension of $\mathrm{T}_{\mathrm{ij}}$. Then, the general equation of motion of cable element is as given follows [21]:

$$
\operatorname{Mu}\left(\mathbf{K}_{L}+\mathbf{K}_{G}\right) \mathbf{u}=\mathbf{0}
$$

where $\mathbf{M}$ is the mass matrix; and $\mathbf{u}$ are the acceleration and displacement vector, respectively; $\mathbf{K}_{\mathbf{L}}$ is the conventional stiffness matrix; KG is the geometric stiffness matrix. They are defined as below.

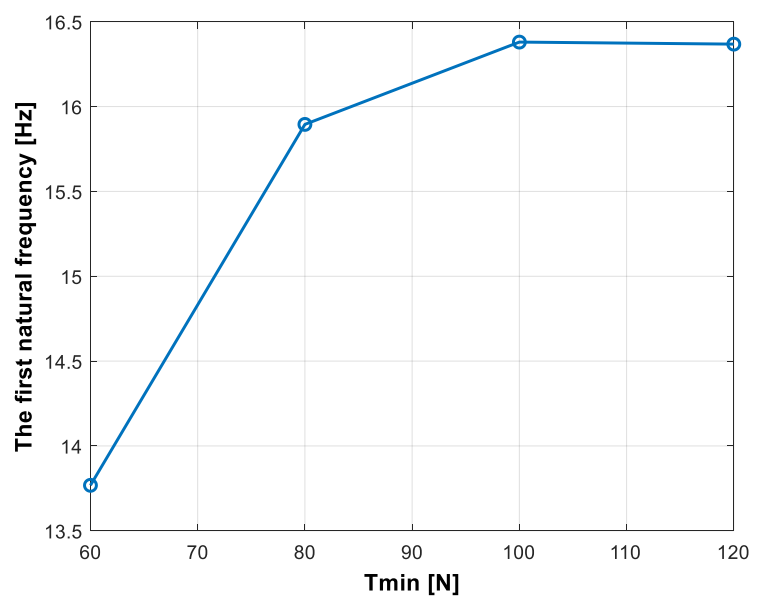

Fig. 6 The dependency of the first natural frequency on values of $T_{\min }$ at the pose of $[0 ; 0 ; 0]$.

$$
\begin{aligned}
& \mathbf{u}=\left[\begin{array}{llllll}
u_{x i} & u_{y i} & u_{z i} & u_{x j} & u_{y j} & u_{z j}
\end{array}\right]^{T} \\
& \mathbf{K}_{L}=\frac{k_{s}-T_{i j}}{l_{i j}}\left[\begin{array}{cc}
\mathbf{G} & -\mathbf{G} \\
-\mathbf{G} & \mathbf{G}
\end{array}\right] ; \mathbf{K}_{G}=\frac{T_{i j}}{l_{i j}}\left[\begin{array}{cc}
\mathbf{I} & -\mathbf{I} \\
-\mathbf{I} & \mathbf{I}
\end{array}\right] \\
& \mathbf{M}=\frac{\gamma_{i j} l_{i j}}{6 g}\left[\begin{array}{cc}
2 \mathbf{I} & \mathbf{I} \\
\mathbf{I} & 2 \mathbf{I}
\end{array}\right]
\end{aligned}
$$

Table 1. The dimension of the proposed CDPR 3D printer frame (Unit: m).

\begin{tabular}{lccccccc}
\hline Position Vector & $x(m)$ & $y(m)$ & $z(m)$ & Position Vector & $x(m)$ & $y(m)$ & $z(m)$ \\
& & & & & & & \\
\hline A1 & 1.25 & 1.25 & 2.5 & A5 & 1.25 & 1.25 & 1.25 \\
A2 & -1.25 & 1.25 & 2.5 & A6 & -1.25 & 1.25 & 1.25 \\
A3 & -1.25 & -1.25 & 2.5 & A7 & -1.25 & -1.25 & 1.25 \\
A4 & 1.25 & -1.25 & 2.5 & A8 & 1.25 & -1.25 & 1.25 \\
\hline
\end{tabular}

Table 2. Parameter values for the free vibration analysis of the proposed CDPR 3D printer.

\begin{tabular}{ll}
\hline Parameters (unit) & Value \\
\hline Mass of the end effector $m(\mathrm{~kg})$ & 30 \\
The cross-sectional areas $A\left(\mathrm{~m}^{2}\right)$ & $50.265 \times 10^{-6}$ \\
The dimensional lengths of the robot's frame $(\mathrm{m})$ & $2.5 \times 2.5 \times 2.5$ \\
The position of the end effector x,y,z $(m)$ & {$[0 ; 0 ; 1.25]$} \\
The modulus of elasticity $E\left(N / m^{2}\right)$ & $2.01 \times 10^{10}$ \\
The weight per unit length $\gamma(N / m)$ & 0.251 \\
The minimum tension $T_{\min }(N)$ & {$[60,80,100,120]$} \\
The maximum tension $T_{\max }(N)$ & 1000 \\
\hline
\end{tabular}




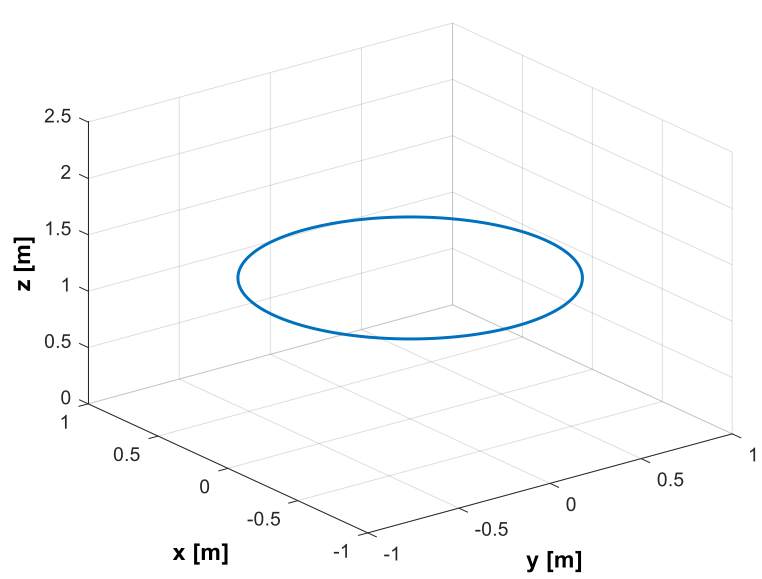

(a)

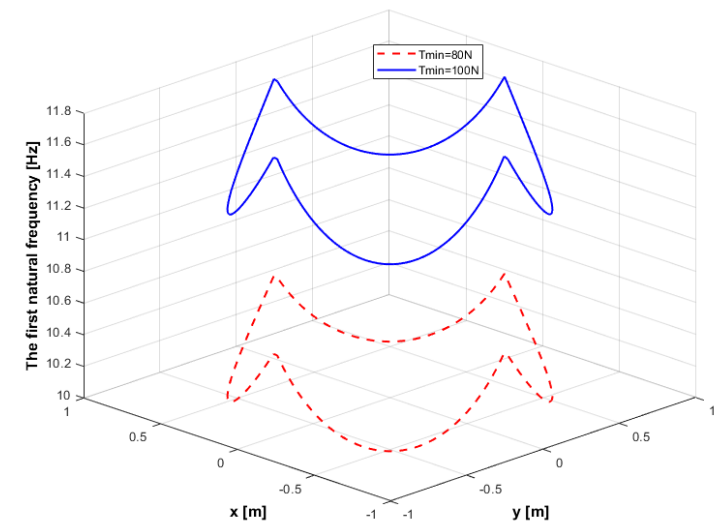

(b)

Fig. 7 (a) The circle trajectory. (b) The first natural frequency of the proposed CDPR 3D printer along the circular trajectory.

Where $\gamma_{i j}$ is the weight per unit length; $\mathrm{k}_{\mathrm{s}}$ is the elastic stiffness with $k_{s}=E A ; \mathbf{G}$ is the transformation matrix which transforms the element stiffness matrix of each cable in the local frame (oxy) to the global frame (OXY) and is given as follows:

$$
\begin{gathered}
\mathbf{G}=\left[\begin{array}{ccc}
l^{2} & m l & n l \\
m l & m^{2} & n m \\
n l & n m & n^{2}
\end{array}\right] \\
l=\left(x_{j}-x_{i}\right) / l_{i j} ; m=\left(y_{j}-y_{i}\right) / l_{i j} ; n=\left(z_{j}-z_{i}\right) / l_{i j}
\end{gathered}
$$

\subsection{Numerical examples}

This section presents a free vibration analysis of the proposed CDPR with two-nozzles. Some assumptions that should be noted for modeling of the proposed CDPR with two-nozzles are given as follows:

- In $3 \mathrm{D}$ printing construction, the printing head moves slowly

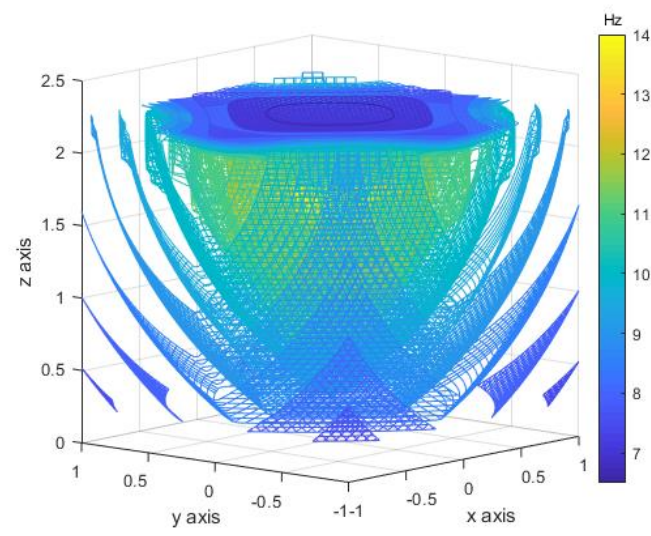

Fig. 8 The first natural frequency of the proposed CDPR $3 \mathrm{D}$ printer in a sub-workspace $(-1 \mathrm{~m} \leq x \leq 1 \mathrm{~m},-1 \mathrm{~m} \leq y \leq 1$ $\mathrm{m}, 0.25 \mathrm{~m} \leq z \leq 2.25 \mathrm{~m}$ ) with $T_{\min }=80 \mathrm{~N}$ and $T_{\max }=1000 \mathrm{~N}$. and the dynamic effect of the printing head on the motion of moving nozzles could be neglected.

- The nozzles are assumed to be fixed to the printing head hence the nozzles and the printing head are considered to be one rigid body.

- All cables are connected at the centroid of the printing head.

- The total mass of the printing head and the additional nozzles is the point mass at the connecting point

- Applied cable tensions are calculated by tension distribution algorithms explained in Section 2.2.

For the free vibration analysis, the general equation of motion for the cable robot's structure can be given as follows:

$\mathbf{M u}^{\mathfrak{Q}}+\mathbf{K u}=\mathbf{0}$

Where, $\mathbf{M}$ and $\mathbf{K}$ are the global mass matrix and the global stiffness matrix, respectively.

The equation for the eigenvalue problem is as follows:

$$
\left(\mathbf{M}-\boldsymbol{\omega}^{2} \mathbf{K}\right) \overline{\mathbf{u}}=\mathbf{0}
$$

Where $\boldsymbol{\omega}$ and $\overline{\mathbf{u}}$ are the natural frequency and the associated displacement vector. To get the nontrivial solutions of the eigenvalue problem, the following determinant must be equal to zero:

$$
\left|\mathbf{M}-\boldsymbol{\omega}^{2} \mathbf{K}\right|=\mathbf{0}
$$

The vibration analysis presented here is implemented by FEM model and is ap plied to the two-nozzle CDPR presented in Fig.1. The dimension and the parameter values used for the analysis are summarized in Table 1 and Table 2. 
Table 3 shows the first ten natural frequencies from the vibration analysis using the FEM model when the minimum tension $T_{\min }$ was set to four different values $60,80,100,120 \mathrm{~N}$. And Fig. 6 shows the relationship of the first natural frequency and the $T_{\min }$ at the pose of $[0 ; 0 ; 0]$. From both Table 3 and Fig. 6 , it is revealed that there is a tendency that the stiffness of the proposed CDPR is increasing with the minimum cable tension $T_{\min }$. For example, when $T_{\min }$ increases from $60 \mathrm{~N}$ to $120 \mathrm{~N}$, the first natural frequency pulls up its values from 13.7 $\mathrm{Hz}$ to $16.4 \mathrm{~Hz}$. Since the stiffness and the vibration resistance of the structure is related to the natural frequency, the stiffness and the vibration resistance of the proposed CDPR 3D printer can be raised by increasing the value of $T_{\min }$.

In Table 3, FEM analysis results were compared with the result of commercial software SAP2000 for the validation. It is observed that the values obtained by FEM analysis are almost identical to the results by SAP2000 thereby the validity of the FEM analysis is now confirmed. Here, it should be pointed out that modeling a CDPR, and making a variation for model change and for other types of CDPRs, using the commercial software requires a lot of time. While the FEM model specifically designed for a CDPR can be easily modified for other comprehensive CDPR models. Also, FEM model provides general formulations of cables for modeling of CDPRs which SAP2000 cannot [5]. Moreover, FEM is more economic in terms of computation time as shown in [5].

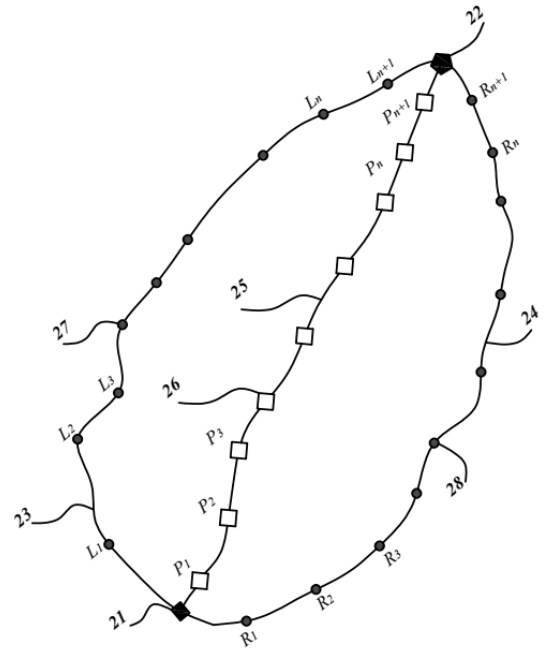

Fig. 9 2D contour of an arbitrary shaped un-symmetric printing part. head.

Fig. 7 (a) shows the circular trajectory for the proposed CDPR 3D printer with a radius of $0.75 \mathrm{~m}$ on the plane $\mathrm{Az}=1.25 \mathrm{~m}$. Fig. 7 (b) illustrates the first natural frequency of the proposed CDPR 3D printer along the circular trajectory with two different values of Tmin. In the case of $\mathrm{T}_{\min }=100 \mathrm{~N}$, at every pose of the trajectory, the proposed CDPR $3 \mathrm{D}$ printer always has the higher first natural frequency than the one in the case of $T_{\min }=80 \mathrm{~N}$. It implies that the stiffness of the proposed

Table 3. Comparison of the first ten natural frequencies of the proposed CDPR 3D printer.

\begin{tabular}{|c|c|c|c|c|c|c|c|c|}
\hline \multirow[t]{3}{*}{$\mathrm{T}_{\min }$} & \multicolumn{2}{|l|}{$60 N$} & \multicolumn{2}{|l|}{$80 \mathrm{~N}$} & \multicolumn{2}{|l|}{$100 \mathrm{~N}$} & \multicolumn{2}{|l|}{$120 \mathrm{~N}$} \\
\hline & Freq & cy (Hz) & Freq & cy (Hz) & Frec & cy (Hz) & Freq & cy $(\mathrm{Hz})$ \\
\hline & 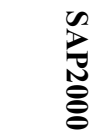 & 武 & 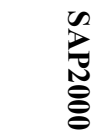 & 전 & 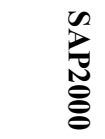 & 武 & 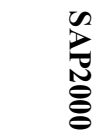 & $\begin{array}{l}\text { 줄 } \\
3\end{array}$ \\
\hline 1 & 13.37 & 13.768 & 15.612 & 15.895 & 15.987 & 16.38 & 16.101 & 16.368 \\
\hline 2 & 13.374 & 13.772 & 15.62 & 15.903 & 15.988 & 16.385 & 16.102 & 16.371 \\
\hline 3 & 13.374 & 13.772 & 15.62 & 15.903 & 16.017 & 16.386 & 16.173 & 16.372 \\
\hline 4 & 13.374 & 13.773 & 15.621 & 15.904 & 16.018 & 16.386 & 16.175 & 16.373 \\
\hline 5 & 13.374 & 13.773 & 15.621 & 15.904 & 16.187 & 16.39 & 16.176 & 16.402 \\
\hline 6 & 13.374 & 13.773 & 15.621 & 15.904 & 16.189 & 16.39 & 16.177 & 16.404 \\
\hline 7 & 13.374 & 13.773 & 15.621 & 15.904 & 16.19 & 16.391 & 16.241 & 16.405 \\
\hline 8 & 13.374 & 13.773 & 15.621 & 15.904 & 16.191 & 16.392 & 16.242 & 16.405 \\
\hline 9 & 15.984 & 16.381 & 15.987 & 16.381 & 17.52 & 17.765 & 19.215 & 19.449 \\
\hline 10 & 15.985 & 16.387 & 15.988 & 16.387 & 17.533 & 17.779 & 19.24 & 19.476 \\
\hline
\end{tabular}




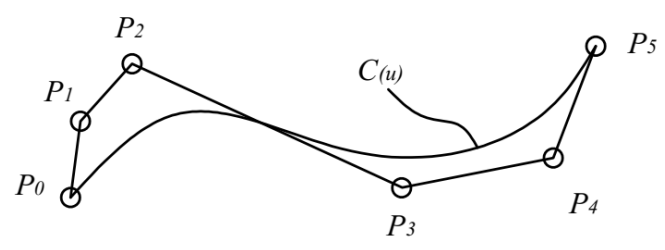

Fig. 10 A perspective view of a non-uniform rational B-spline (NURBS) of the optimal path for the printing head.

CDPR 3D printer can be improved by increasing the values of Tmin.

Fig. 8 illustrates the first natural frequency of the proposed CDPR 3D printer for different positions in 3D space. From the figure, it is shown that as the printing head moves up, its natural frequency gets increased and at the center of the workspace, the natural frequency reaches its maximum. Based on this observation, it is inferred that the structural stiffness of the CDPR at a low position in the workspace is low and vulnerable to vibration. In contrast, if the end-effector moves towards the top center of the workspace, the structural stiffness increases thus vibration resistance is achieved.

\section{Path optimization}

For a two-nozzle CDPR, the path optimization of the printing head is conducted based on three constraints. (1) minimize the path lengths difference between two nozzles in the printing process to balance the required motion for two nozzles; (2) achieve the shortest path length to save the printing time; (3) the path shall be smooth based on the nonuniform rational basis spline (NURBS).

\subsection{The objective function for path optimization}

Fig. 9 shows the 2D contour of an arbitrary shape of the asymmetric part. As mentioned, to print this part, an optimal path of the printing head of Fig. 1 must be determined ahead with three following criteria being satisfied: (1) the path for the printing head should line in the middle of the contour to save and balance the time required to move both nozzles. This means that the difference between the path lengths of two nozzles shall be minimized and both nozzles will travel as similar length as possible; (2) The path length of the printing head shall be minimum to save the printing time; (3) The path shall be as smooth as possible by using a NURBS curve.

In Fig. 9, the contour is constructed with a set of discretized pointsthe left contour 23 consisting of discretized points 27 of $\mathrm{L}_{1}, \mathrm{~L}_{2}, \ldots, \mathrm{L}_{\mathrm{n}}$ and the right contour 24 consisting of discretized points 28 of $R_{1}, R_{2}, \ldots$, $\mathrm{R}_{\mathrm{n}}$. The optimal path 25 of the printing head should go through the start point 21, target point 22 and inside points $\mathrm{P}_{1}, \mathrm{P}_{2}, \ldots, \mathrm{P}_{\mathrm{n}}$, and it should satisfy the first two conditions of the optimal path.

With the given conditions, the optimal path of the printing head can be

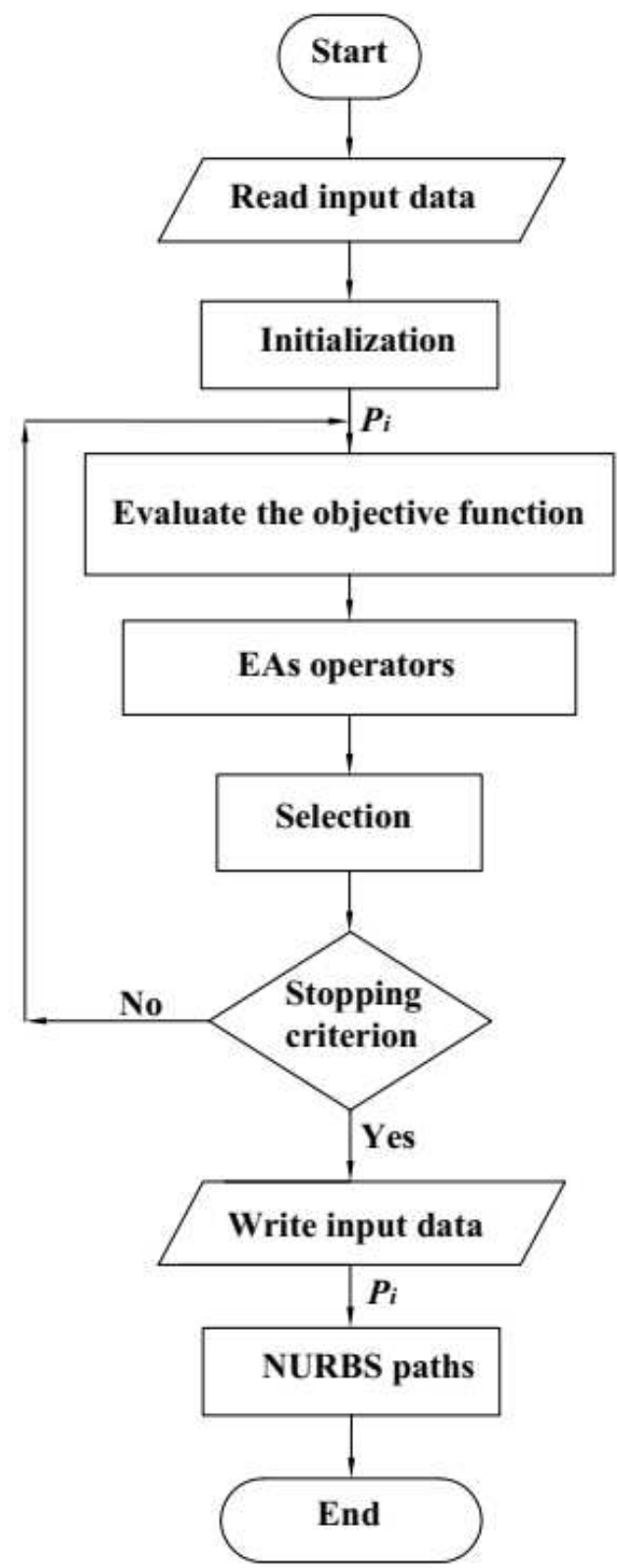

Fig. 11 The flow chart of path optimization.

determined by solving the optimization problem minimizing the following objective function:

$$
f_{\text {path }}=\alpha \sum_{i=1}^{n-2}\left|\left(\left\|\vec{P}_{i} L_{i}\right\|-\left\|\vec{P}_{i} R_{i}\right\|\right)\right|+\sum_{i=1}^{n}\left\|\vec{P}_{i+1} P_{i}\right\|
$$

Where, $n$ - numbers of discretized points (which are defined by users),

Table 4. Parameters for path optimization in Example 1.

\begin{tabular}{lllllllll}
\hline & $\boldsymbol{G E}$ & $\boldsymbol{N P}$ & $\boldsymbol{C R}$ & $\boldsymbol{M U}$ & $\boldsymbol{S E}$ & $\boldsymbol{\theta}$ & $\boldsymbol{\varepsilon} 1$ & $\boldsymbol{\varepsilon} 2$ \\
\hline GA & 100 & 100 & - & 0.2 & 0.5 & - & - & - \\
DE & 100 & 100 & 0.5 & - & - & - & - & - \\
PSO & 100 & 100 & - & - & - & 0.6 & 2 & 2 \\
\hline
\end{tabular}


Table 5. Coordinates of boundary points.

\begin{tabular}{cccc}
\hline & Left boundary & \multicolumn{2}{c}{ Right boundary } \\
\hline$\underline{\boldsymbol{x}}$ & $\underline{\boldsymbol{y}}$ & $\underline{\boldsymbol{x}}$ & $\underline{\boldsymbol{y}}$ \\
\hline-6 & -4 & 1 & -4.5 \\
-8 & -2.5 & 3 & -3 \\
-9 & 0 & 5 & -2 \\
-5 & 3 & 5 & 1.5 \\
-5.5 & 6 & 5 & 5 \\
-3.5 & 8 & 4 & 6.5 \\
-1.5 & 10 & 3 & 10 \\
\hline
\end{tabular}

Table 6. The optimal points.

\begin{tabular}{ccccccc}
\hline \multirow{2}{*}{ Solutions } & \multicolumn{2}{c}{ DE } & \multicolumn{2}{c}{ GA } & \multicolumn{2}{c}{ PSO } \\
\cline { 2 - 7 } & $\underline{\boldsymbol{x}}$ & $\underline{\boldsymbol{y}}$ & $\underline{\boldsymbol{x}}$ & $\underline{\boldsymbol{y}}$ & $\underline{\boldsymbol{x}}$ & $\underline{\boldsymbol{y}}$ \\
\hline $\boldsymbol{P}_{1}$ & -2.499 & -4.222 & -2.509 & -4.126 & -2.496 & -4.301 \\
$\boldsymbol{P}_{2}$ & -2.497 & -2.861 & -2.494 & -2.912 & -2.501 & -2.721 \\
$\boldsymbol{P}_{3}$ & -2.005 & -0.99 & -2.031 & -0.931 & -2.059 & -0.585 \\
$\boldsymbol{P}_{4}$ & -0.042 & 2.561 & -0.092 & 2.918 & -0.067 & 2.696 \\
$\boldsymbol{P}_{5}$ & -0.239 & 5.384 & -0.228 & 5.297 & -0.234 & 5.334 \\
$\boldsymbol{P}_{6}$ & 0.214 & 7.493 & 0.215 & 7.424 & 0.267 & 7.166 \\
$\boldsymbol{P}_{7}$ & 0.749 & 10 & 0.762 & 10 & 0.75 & 10 \\
\hline
\end{tabular}

Table 7. Distances from the optimal path to the left and right half contour for all three algorithms.

\begin{tabular}{ll|ll|ll}
\hline \multicolumn{2}{c|}{ DE } & \multicolumn{2}{c|}{ GA } & \multicolumn{2}{c}{ PSO } \\
\hline $\begin{array}{l}\text { Distances to } \\
\text { the left half } \\
\text { contour }\end{array}$ & $\begin{array}{l}\text { Distances to } \\
\text { the right half co } \\
\text { ntour }\end{array}$ & $\begin{array}{l}\text { Distances to } \\
\text { the left half } \\
\text { contour }\end{array}$ & $\begin{array}{l}\text { Distances to } \\
\text { the right half co } \\
\text { ntour }\end{array}$ & $\begin{array}{l}\text { Distances to } \\
\text { the left half } \\
\text { contour }\end{array}$ & $\begin{array}{l}\text { Distances to } \\
\text { the right half co } \\
\text { ntour }\end{array}$ \\
\hline 3.508 & 3.510 & 3.493 & 3.529 & 3.517 & 3.502 \\
5.515 & 5.499 & 5.521 & 5.495 & 5.503 & 5.508 \\
7.065 & 7.077 & 7.031 & 7.112 & 6.966 & 7.199 \\
4.977 & 5.152 & 4.909 & 5.286 & 4.942 & 5.206 \\
5.297 & 5.253 & 5.319 & 5.236 & 5.308 & 5.245 \\
3.748 & 3.914 & 3.759 & 3.896 & 3.858 & 3.792 \\
2.249 & 2.251 & 2.262 & 2.238 & 2.250 & 2.250 \\
\hline
\end{tabular}

$P_{i}$ is the ith inside points; $L_{i}$ is the ith node on the left boundary; $R_{i}$ is the ith node on the right boundary; $\alpha$ is the penalty parameter and set to be $10^{2}$ to ensure two parts in Eq.(19) are equally weighted.

\subsection{NURBS interpolation for paths}

For the optimal path to satisfy the third condition - the smoothness, a
NURBS is used considering its high level of flexibility and great capacity to produce natural smooth curves. In addition to such features, NURBS is often used for the optimal path generation as it has ability to represent the geometrical shapes in a compact form as reported in [18]. NURBS of which schematic view is shown in Fig. 10 and is 


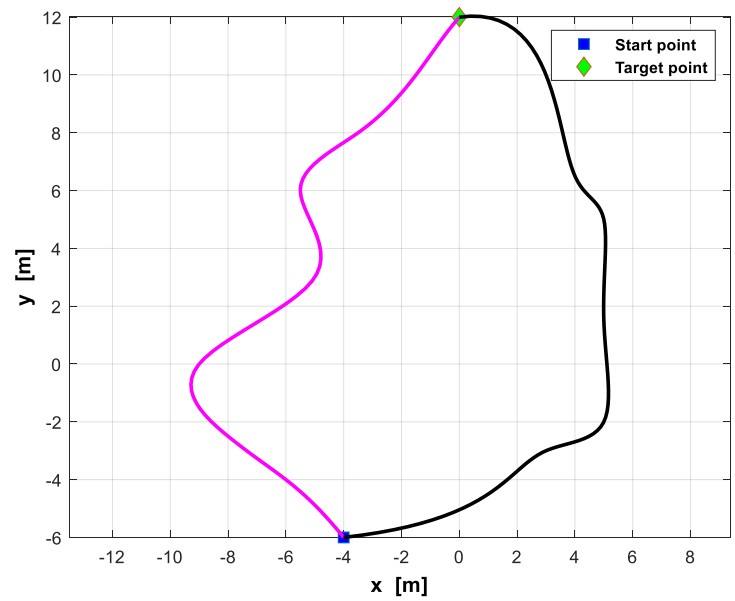

Fig. 12 The shape of the printing object in Example 1.

defined as Eq. (20) is used for the optimal path:

$$
C(u)=\sum_{i=0}^{n} R_{i, p}(u) P_{i} \quad a \leq u \leq b
$$

where $\left\{P_{i}\right\}$ are the $n$ control points $(n>2) ;\left\{R_{i, p}(u)\right\}$ are the rational B-spline basis functions and is defined as follows:

$$
R_{i, p}(u)=\frac{N_{i, p}(u) \omega_{i}}{\sum_{j}^{n} N_{j, p}(u) \omega_{j}}
$$

$N_{i, p}(u)$ denotes the $i^{\text {th }} \mathrm{B}$-spline basis function of parameter $u$ and degree $p, \omega_{i}$ stands the weights of control points.

The optimization problem to find the inside points which compose an optimal printing head path is solved by evolutionary algorithms such as GA, DE, and PSO which are the most-used evolutionary algorithms in engineering optimization and path optimization. The sequence of path optimization is shown in the flow chart of Fig. 11.

A numerical simulation is performed in Section 4.3 to show the feasibility of GA, DE and PSO in finding the optimal path of the multinozzle CDPR.

\subsection{Computer simulation of path planning}

In the following simulation, we assume that the initial and target points are already determined. The main objective is to find the optimal paths that have a minimum length. To find out the points of the optimal paths, genetic algorithm (GA), particle swarm optimization (PSO) and differential evolution (DE) are used. Parameters for these algorithms are provided in Table 4 . Where GE is the number of generations, $N P$ is the number of populations, $C R$ is the crossover probability, $M U$ is the fraction of mutation, $S E$ is the percentages of selection, $\theta$ is the inertia factor and is equal to 0.6 , and $\varepsilon_{l}$ and $\varepsilon_{2}$ are learning parameters or acceleration constants, respectively and are set to be 2 [22].

Also, NURBS curves in which all weights of control points set to one

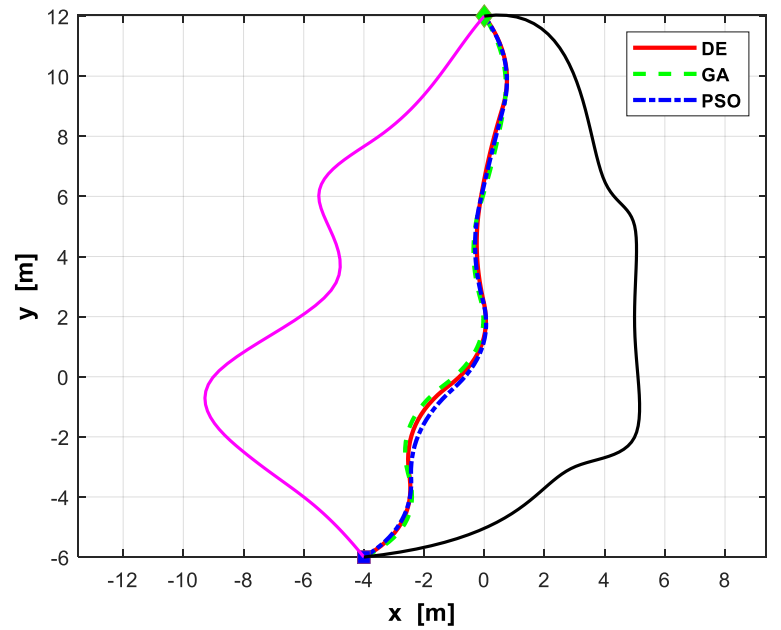

Fig. 13 The optimal path obtained by DE, GA and PSO.

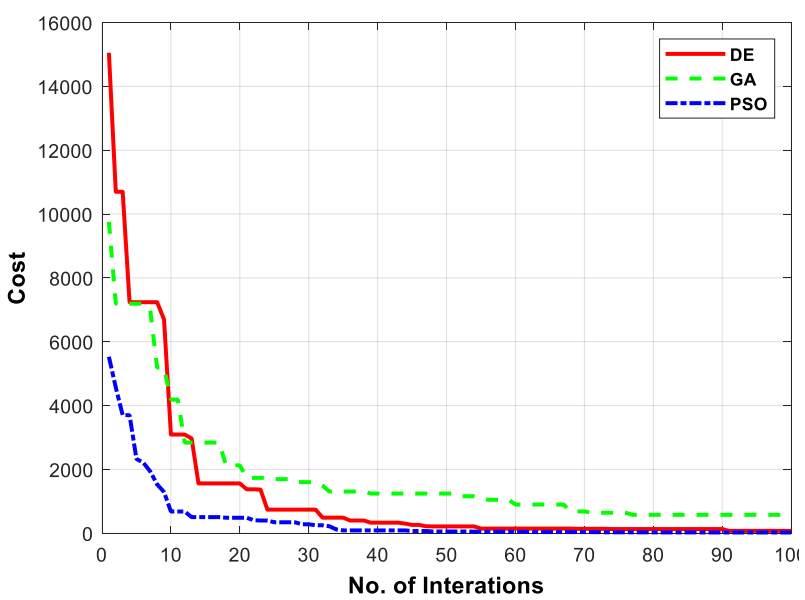

Fig. 14 The convergence cost of DE, GA and PSO.

are used for the optimal paths of the printing head of a two-nozzle CDPR 3D printer [18].

Let us consider an arbitrary shaped building shown in Fig. 12. The contour of the building to be printed is divided into two sides, the leftand right-half contours. The coordinates of 7-discretized points along these contours are given in Table 5 .

The path optimization by GA, PSO, and DE is implemented using Matlab R2018b program on a desktop computer with Intel Core(TM) i7-7700 CPU@3.60GHz (8 CPUs), 8.00 GB RAM of memory, and Windows 10 Professional with 64-bit operating system.

The optimal points computed by GA, PSO, and DE are shown in Table 6. And the optimal paths obtained by NURBS based on those points are shown in Fig. 13. From the figure, it is confirmed that three algorithms generated almost similar optimal paths and the optimal paths are not only located in the middle of the printing parts but also forms the shortest paths. Note that the smoothness requirement of paths is satisfied by using NURBS curves.

Table 7 shows the travel distances of both nozzles obtained from those three algorithms and it confirms that the optimal path is located in the 
middle. Since they travel almost same distances, the speed of two nozzles would be quite similar. The similar speed patterns of both nozzles help suppress the possible vibration that could be incurred when the nozzle speed on both sides unbalances and large acceleration/deceleration is required to compensate the speed unbalance.

For the comparison of the effectiveness of the algorithms, convergence rate of the fitness functions of those algorithms are illustrated Fig. 14. From the figure, PSO and DE require a shorter computation time than GA, hence they fit better for the real time application.

From the above results, evolutionary algorithms such as GA, DE and PSO are validated to be effective for path planning of multi-nozzle CDPRs.

\section{Conclusion}

A novel multi(two)-nozzle CDPR 3D printer is proposed for the reduction of printing time and the construction cost. The kinematics and dynamics of the printing head and nozzles were analyzed. Due to slow and steady motion of the printing head in $3 \mathrm{D}$ printing construction, dynamic effect of the nozzles on the printing head was neglected. For the path generation of the two-nozzle printing head, three constraintsminimizing the path lengths difference between the two nozzles; keeping the path length shortest to save the printing time; maintain the smoothness of the path using the NURBS-were imposed. Three evolutionary algorithms (GA, DE, PSO) were applied for the path optimization problem having above mentioned constraints and they successfully generated shortest path which is suitable to control both nozzles mounted on the printing head.

To investigate the vibration of the two-nozzle CDPR, its natural frequencies were computed by using the finite element method and its results were verified with a comparison with the commercial software results. Through natural frequency analysis, it was found that the higher stiffness or the higher natural frequencies could be achieved by increasing the minimum cable tension constraint used in the tension distribution optimization algorithm. It is also revealed that the natural frequency goes up as the printing head moves up towards the top center of the workspace.

Acknowledgements The authors would like to acknowledge the financial support from the Ministry of Education and Science Technology of the Korean government.

Author contribution Sy Nguyen-Van: formal analysis, investigation, software, writing-original draft, visualization. Kwan-Woong Gwak: data curation, funding acquisition, methodology, project administration, resources, review, and editing.

Funding This research was supported by a grant (NRF2020R1A4A2002855) from NRF (National Research Foundation of Korea) funded by MEST (Ministry of Education and Science Technology) of Korean government

Availability of data and material For reasonable request, the datasets of the current study are available from the corresponding author.

Code availability Not applicable.

\section{Declarations}

Conflict of interest The authors declare no competing interests.

Ethics approval Not applicable.

Consent to participate Not applicable.

Consent for publication Not applicable

\section{References}

[1] A. Pott, Cable-Driven Parallel Robots: Theory and Application. Springer International Publishing, 2018.

[2] E. Barnett and C. Gosselin, "Large-scale 3D printing with a cable-suspended robot," Addit. Manuf., vol. 7, pp. 27-44, 2015.

[3] J.-B. Izard et al., "Large-scale 3D printing with cable-driven parallel robots," Constr. Robot., vol. 1, no. 1-4, pp. 69-76, 2017.

[4] P. Bosscher, R. L. Williams, L. S. Bryson, and D. CastroLacouture, "Cable-suspended robotic contour crafting system," Autom. Constr., vol. 17, no. 1, pp. 45-55, 2007.

[5] S. Nguyen-Van, K. W. Gwak, D. H. Nguyen, S. G. Lee, and B. H. Kang, "A novel modified analytical method and finite element method for vibration analysis of cable-driven parallel robots," J. Mech. Sci. Technol., no. September, 2020.

[6] Y. W. D. Tay, B. Panda, S. C. Paul, N. A. Noor Mohamed, M. J. Tan, and K. F. Leong, "3D printing trends in building and construction industry: a review," Virtual Phys. Prototyp., vol. 12, no. 3, pp. 261-276, 2017, doi: $10.1080 / 17452759.2017 .1326724$

[7] P. Raja, "Optimal path planning of mobile robots: A review," Int. J. Phys. Sci., vol. 7, no. 9, pp. 1314-1320, 2012, doi: 
10.5897/IJPS11.1745.

[8] A. Atyabi, "Review of classical and heuristical navigation and path planning approaches Classical Methods," Int. J. Adv. Comput. Technol., vol. 5, no. January, pp. 1-17, 2013.

[9] R. Toogood, Hong Hao, and Chi Wong, "Robot path planning using genetic algorithms," pp. 489-494, 2002, doi: 10.1109/icsmc.1995.537808.

[10] Q. C. Nguyen, Y. Kim, and H. Kwon, "Optimization of layout and path planning of surgical robotic system," Int. J. Control. Autom. Syst., vol. 15, no. 1, pp. 375-384, Feb. 2017, doi: 10.1007/s12555-015-0418-z.

[11] Woong-Gie Han, Seung-Min Baek, and Tae-Yong Kuc, "Genetic algorithm based path planning and dynamic obstacle avoidance of mobile robots," in 1997 IEEE International Conference on Systems, Man, and Cybernetics. Computational Cybernetics and Simulation, 2002, pp. 27472751, doi: 10.1109/icsmc.1997.635354.

[12] A. K. Pamosoaji, M. Piao, and K.-S. Hong, "PSO-based Minimum-time Motion Planning for Multiple-vehicle Systems Considering Acceleration and Velocity Limitations," Int. J. Control. Autom. Syst., Apr. 2019, doi: 10.1007/s12555018-0176-9.

[13] E. Masehian and D. Sedighizadeh, "Multi-objective PSObased algorithms for robot path planning," Adv. Electr. Comput. Eng., vol. 10, no. 4, pp. 69-76, 2010, doi: 10.4316/aece.2010.04011.

[14] J. Chakraborty, A. Konar, L. C. Jain, and U. K. Chakraborty, "Cooperative multi-robot path planning using differential evolution,” J. Intell. Fuzzy Syst., vol. 20, no. 1-2, pp. 13-27, 2009, doi: 10.3233/IFS-2009-0412.

[15] X. Zhang, J. Chen, B. Xin, and H. Fang, Online path planning for UAV using an improved differential evolution algorithm, vol. 44, no. 1 PART 1. IFAC, 2011.

[16] A. K. Singh, A. Aggarwal, M. Vashisht, and R. Siddavatam, "Robot motion planning in a dynamic environment using offset Non-Uniform Rational B-Splines (NURBS)," in Proceedings of the IEEE International Conference on Industrial Technology, 2011, pp. 312-317, doi: 10.1109/ICIT.2011.5754393.

[17] X. Shi, H. Fang, and L. Guo, "Multi-objective optimal trajectory planning of manipulators based on quintic NURBS," in 2016 IEEE International Conference on Mechatronics and Automation, IEEE ICMA 2016, 2016, pp. 759-765, doi: 10.1109/ICMA.2016.7558658.

[18] S. Jalel, P. Marthon, and A. Hamouda, "A new path generation algorithm based on accurate NURBS curves," Int. J. Adv. Robot. Syst., vol. 13, no. 2, pp. 1-14, 2016, doi: $10.5772 / 63072$.

[19] X. Diao and O. Ma, "Vibration analysis of cable-driven parallel manipulators," Multibody Syst. Dyn., vol. 21, no. 4, pp. 347-360, 2009.

[20] R. Babaghasabha, M. A. Khosravi, and H. D. Taghirad, "Robust PID control of fully-constrained cable driven parallel robots," Mechatronics, vol. 24, no. 2, pp. 87-97, 2014.

[21] M. L. Gambhir and B. de V. Batchelor, "Finite element study of the free vibration of 3-D cable networks," Int. J. Solids Struct., vol. 15, no. 2, pp. 127-136, 1979.

[22] X. S. Yang, Nature-Inspired Optimization Algorithms. Elsevier, 2014. 